\title{
Study on the Role Changes of Students in Blended Learning Based on BYOD
}

\author{
Yulei Gao \\ International Tourism Department, Nanjing Institute of Tourism and Hospitality \\ Nanjing, China \\ allen.0403@163.com
}

Keywords: role changes, blended learning, BYOD.

\begin{abstract}
BYOD has become one of the most important trends in educational informatization worldwide. In this context, in order to help students to adapt to this change as soon as possible and improve teaching efficiency, six role changes of students in blended learning are proposed, that is, self-managers of BYOD, participants in teaching design, selectors of online information, constructors of knowledge meaning, quantified selfers of the learning process and implementers of teaching evaluation.
\end{abstract}

\section{基于BYOD的混合式学习中学生角色的转变研究}

\author{
高玉垒 \\ 南京旅游职业学院国际旅游系, 南京, 江苏, 中国 \\ allen.0403@163.com
}

关键词: 角色转变; 混合式学习; BYOD

中文摘要. 自带设备 (BYOD) 已经成为全球教育信息化的重要发展趋势之一。在此背景下, 为帮助学生尽快适应这一变化, 提高教学效率, 提出了基于BYOD的混合式学习中学生角色 六大转变, 即BYOD的自我管理者、教学设计的参与者、网络信息的䇥选者、知识意义的建 构者、学习过程的自我量化者和教学评价的实施者。

\section{1. 引言}

进入21世纪, 随着信息技术的飞速发展, 人们的学习和工作方式发生了翻天覆地的变化。 其典型表现一是混合式学习模式的出现, 二是 “自带设备” (即BYOD, 英文Bring Your Own Device的首字母缩写) 的兴起。混合式学习是由于 20 世纪90年代末以来在线学习 (E-learning) 的局限性日益突出, 在企业培训领域应运而生的一种学习模式, 并逐渐吸引了教育界的关注。 2009年，BYOD起源于IT企业，主要指员工将自己的笔记本电脑接入企业网络以支持自己的 工作, 提高工作效率。这种模式也很快被引入到教育教学领域, 师生通过BYOD获取教学资 源、进行信息交流、开展教学活动, 以提高学生的学习积极性和学习效果。

在网络技术进入移动互联网时代后，混合式学习和BYOD的优势更加明显，而且两者的 结合深刻地变革着传统的教育教学模式。但随之而来的一个问题是, 习惯了坐在教室里听老 师讲课的学生很难适应这种变化, 除了听课感觉无所适从。究其原因, 主要是从传统的教师 讲授为主的教学模式向基于BYOD的混合式学习转型过程中, 学生对自己的角色定位不清晰。 基于此, 本文论述了新型教学模式中学生角色转变的六大方向。 


\section{2. 基于BYOD的混合式学习中学生角色的转变}

移动互联网时代, 学生可以通过BYOD随时随地获取大量的学习资源, 其学习场域和学 习方式发生了重大变化, 加之社会对人才能力的需求变化, 打破了学生传统教学模式中听讲 的单一角色。学生必须适应迅速变化的时代, 作为学习者, 其角色身份开始变的多元, 在学 习的不同阶段扮演着不同的角色，内涵更加丰富。

\subsection{BYOD的自我管理者}

开展混合式学习离不开学习管理系统 (LMS, Learning Management System) 的支持。因 此, BYOD进课堂成为当今教育发展变化的重要趋势之一。移动互联网时代, BYOD更多的 表现为智能手机, 它不仅具备笔记本电脑的基本功能, 而且小巧、携带方便, 更重要的是交 际沟通方便。当代大学生是移动互联网时代的原住民, 智能手机成为他们生活中的标配。“机 不离手” 成为大部分学生的真实写照, 反映在课堂上就是出现了 “低头族”。课堂手机问题 俨然已经成为一个 “国际难题” ，因此各个高校纷纷发起 “无手机课堂” 倡议。这显然是与 当今教育发展趋势相悖的。一味的 “奢” 不仅是治标不治本, 而且还会引起学生的反感。因 此, 必须采取 “疏” 的策略, 教师充分利用学生的BYOD开展丰富的教学活动, 引导学生合 理利用手机。

学生是学习活动的主体。根据马克思主义的哲学观, 学生是内因, 教师的引导必须通过学 生才能起作用。因此, 面对BYOD的巨大诱惑, 学生必须转变观念, 认识到自己才是学习的 主人, 而BYOD是辅助学习的有力工具。另外, 学生应该积极参与教师利用BYOD开展的各 项教学活动, 减少课堂上对设备其他功能的应用。最后, 学生可以自己建立课堂使用BYOD 的约束机制, 或使用番茄工作法类App强制约束自己合理利用, 逐渐形成良好的习惯。有效 的BYOD自我管理者角色是开展混合式学习的重要前提条件。

\section{2 教学设计的参与者}

传统的教学模式中以教师为中心, 教师掌握着教学活动的主动权, 学生处于被支配的地 位, 是课堂的被动参与者。这种模式直接导致了教学效率低下, 教师教的累, 学生学的也累。 这一问题突出反映在教学分析、教学活动设计、教学实施以及教学评价等教学设计的各个环 节上。究其原因, 从主观上来讲, 教师长期受行为主义教学理论的影响, 对整个教学拥有绝 对的把控权, 教师主要从个人经验出发进行教学设计, 忽视了学生的因素, 学生只能被动的 参与课堂学习; 从客观上讲, 由于时间、技术等条件限制, 教师使学生参与教学设计的成本 与代价比较高。很显然, 这种做法的弊端是比较多的, 如无法真实了解学生的知识准备、学 习需求与学习效果。

随着现代教学理论和建构主义的发展, 人们开始对传统的教学模式不断地进行抨击与改 进。改变传统的教学模式, 重要的一步就是吸引学生参与到教学设计中来。移动互联网环境 下BYOD的发展为学生参与教学设计提供了极大便利。首先, 在教学分析阶段, 教师可以通 过编制调查问卷通过在线教学平台向学生发放并收集数据, 这为了解学生、针对性地开展教 学提供了基础; 其次, 在教学内容方面, 每个学生对知识的需求不同, 他们有权参与决定应 该学生, 一方面学生可以通过BYOD 向老师表达自己对教学内容的需求, 另一方面, 学生需 求不同了, 则必须开展混合式教学才能满足这种个性化发展; 再次, 学生应该参与到教学活 动的设计中, 与教师共同决定什么内容适合在线自主学习, 什么内容需要线下通过集中教授 学习? 线下的面对面学习中, 应该是听教师讲授, 还是个人独自探究, 抑或小组合作探究? 这些活动的设计应该吸引学生参与设计, 才能充分调动学生的积极性; 最后, 教学评价的设 计也需要有学生的参与。学生可以与老师讨论决定对某项教学活动如何开展教学评价。不管 是自评、教师评价还是学生互评, 学生都应该参与到评价标准与内容的设计中。提高评价的 针对性, 学生才能对学习成效产生预期。教学设计的这些环节在传统教学环境下是很难实现 
的, 信息技术却很容易地解决了这些问题。因此, 开展基于BYOD的混合式学习, 促进学生 发展, 必须使学生由一个听讲者向一个教学设计参与者转变。

\section{3 网络信息的笁选者}

传统环境下教学中, 教师是知识的权威和主要甚至是唯一来源, 学生主要通过教师获取 知识。但进入 21 世纪, 信息技术飞速发展, 海量信息资源充满着网络, 教师的知识权威地位 受到威胁。学生可以通过网络获取大量的学习资源。但随之而来的问题是, 越是资源丰富, 学生越是难以选择适合自己水平和需求的信息。同时, 网络资源质量良莠不齐, 甚至许多信 息存在着严重的错误或观点偏颇。因此, 学生必须做一个网络时代的信息篮选者, 选择对自 己有用的信息。

学生有效的筷选网络信息资源, 除了国家加大网络监管力度和教师引导等外界环境之外, 自身还需要不断提升信息笁选能力。一方面学生应该熟悉自己学科常用的信息资源网站, 这 些内容是对自己专业学习最有帮助的, 另一方面, 学生需要提高信息概括能力, 学会用关键 词等字段通过搜索引擎进行查找。最后, 学生作为BYOD的拥有者, 在通过BYOD帮助自己 学习的同时也应该注意网络信息安全, 不轻信他人传播的信息, 自己也不能传播未经证实的 各种消息、谣言。

\section{4 知识意义的建构者}

在早期的学习理论影响下, 学习的过程就是教师向学生传递知识的过程, 学生是信息被 动接收者。但英语纽卡斯尔大学教授苏伽特 - 米特拉（Sugata Mitra）在印度的“墙中洞”实验 (Hole in the Wall) 却证明了建构主义学习观的正确性。建构主义学习观认为, 学习是学生在 一定的情境中, 借助他人的帮助, 通过意义建构而获取知识的过程。因此, 学习是一个主动 的过程, 学生对外部信息进行主动的选择和加工, 以自己的原有知识经验为基础, 对新的信 息进行意义建构和理解。

基于BYOD的混合式学习改变了传统的教师单向传授知识的学习过程, 理想的学习环境 应该包括情境、协作、交流和意义建构四个部分。因此, 新的环境下, 教师更多的是为学生 创设情境, 引导学生进行利用BYOD搜集信息, 组织学生间的协作交流进而构建自己的知识 体系。在这一过程中, 学生逐渐地向一个知识意义的建构者角色转变。

\section{5 学习过程的量化自我者}

量化自我的概念最早由美国著名科技杂志《连线》（WIRED）的两位记者盖瑞・伍尔夫 (Gary Wolf) 和凯文・凯利（Kevin Kelly）提出的。期初主要应用与医学健康领域, 指人们 通过可穿戴设备及移动终端实时记录个人生命数据, 并通过数据反馈进行自我调整。后来, 这一迅速应用与教育领域。对于学生的学习来说, 量化自我有利于学生了解自己的学习状况, 明确自己学习的优势与不足, 进而帮助学生发挥优势, 弥补学习上的不足。

量化自我离不开大数据的支持。学生在基于BYOD的混合式学习中, 通过学习平台留下 了大量学习数据, 包括资源学习、活动参与、学习成绩等。学生可以通过这些数据找出自己 做的好的与差的方面, 好的方面应该继续保持, 差的方面就是学习短板, 应该加倍努力克服。 此外, 学生还可以通过这些数据进行个人学习的历时比较, 了解自己学习进步状况; 也可以 进行个人与其他同学的横向比较, 找出与其他人的差距, 虚心请教优秀的同学, 促使自己获 得进步。

\section{6 教学评价的实施者}

教学评价是衡量教学效果, 促进教学改革的重要手段, 因此教学评价应当尽量做到全面、 科学、客观。但在传统的教学模式中, 教学评价的问题主要反映在不全面上, 如只注重教师 
评价、终结性评价和学习成绩, 而较少关注学生自评、过程性评价和学生能力评价, 因此也 有“一考定终身”的说法。要改变这一现象, 就要从评价主体、评价方式等方面进行改革。

在基于BYOD的混合式学习中, 学生是教学设计的重要参与者, 参与了教学评价的设计, 同时也教学评价的实施者。首先, 学生可以通过学习平台的大数据进行总体自我评价, 也可 以就自己的某项作业任务根据评分点进行自我评价; 其次, 学生可以在教学平台对其他的作 业任务进行实名或匿名互评; 最后, 学生也可以对教师的教学进行评价, 以利于教师及时调 整教学策略。评价既可以是客观性的分数评价, 也可以是主观性的评语评价, 无论通过何种 方式, BYOD都可以方便使师生获得及时的反馈, 改进教学。

\section{3. 结束语}

BYOD已成为全球教育信息化的重要趋势之一，而混合式学习也当今最流行的教学模式。 两者的结合势必会对传统的教学模式产生颠覆性变革。就学生而言, 必须要改变传统的课堂 听讲者角色, 尽早适应基于BYOD的混合式学习者的角色。一方面, 这有利于提高学生的学 习积极性和主动性, 提高学习效果; 另一方面, 学习的过程是学生在师生协助下自我建构知 识意义的过程, 这有利于学生学会学习, 培养自主学习和终身学习的能力。

\section{致谢}

本文为江苏省教育科学“十三五”规划青年教师专项重点资助课题“基于BYOD的高职英 语混合式学习研究与实践”的阶段性成果，课题批准文号：C-a/2016/03/04。

\section{References}

[1] G. Gu, The challenge and choice of vocational school teachers in the "Internet+"era: role, thinking transformation and development path, Vocational and Technical Education, vol.36, pp. 32-37, 2017.

[2] Y. Gao, A survey on higher vocational college students' mobile English reading in the context of big data, Digital Education, vol.18, pp. 47-51, 2017.

[3] $\mathrm{X} . \mathrm{Wu}$, The instructional design for the online participative teaching, Modern Educational Technology, vol.19, pp. 66-69, 2009.

[4] P. Wen and G. Jia, Constructionism theory \& teaching reform---a summary of constructionism learning theory, Theory and Practice of Education, vol. 22, pp. 17-22, 2002.

[5] J. Wang, J. Yan and Y. Wang, A theoretical perspective and enlightenment of the "hole in the wall” experiment, e-Education Research, vol. 261, pp. 5-8, 28, 2015.

[6] R. Chen and C. Yang, Quantified self: new opportunities of education research in the big data era---The 2014 Horizon Report of NMC, Modern Educational Technology, vol.24, pp. 5-11, 2009.

[7] Z. Ma, X. Wang and Q. Long, A literature review of online peer assessment, Journal of Distance Education, vol.223, pp. 86-92, 2014.

[8] H. Zuo and F. Huang, The essay on the shift of the role of student:from learning subject to knowledge subject, Research in Educational Development, vol.6 pp. 68-74, 84, 2013. 